\title{
Self-Generated Magnetic Fields in the Stagnation Phase of Indirect-Drive Implosions on the National Ignition Facility
}

\begin{abstract}
C. A. Walsh, ${ }^{*}$ J. P. Chittenden, K. McGlinchey, N. P. L. Niasse, ${ }^{\dagger}$ and B. D. Appelbe
Blackett Laboratory, Imperial College, London SW7 2AZ, United Kingdom

(Received 8 December 2016; revised manuscript received 17 March 2017; published 10 April 2017)

Three-dimensional extended-magnetohydrodynamic simulations of the stagnation phase of inertial confinement fusion implosion experiments at the National Ignition Facility are presented, showing selfgenerated magnetic fields over $10^{4} \mathrm{~T}$. Angular high mode-number perturbations develop large magnetic fields, but are localized to the cold, dense hot-spot surface, which is hard to magnetize. When low-mode perturbations are also present, the magnetic fields are injected into the hot core, reaching significant magnetizations, with peak local thermal conductivity reductions greater than $90 \%$. However, Righi-Leduc heat transport effectively cools the hot spot and lowers the neutron spectra-inferred ion temperatures compared to the unmagnetized case. The Nernst effect qualitatively changes the results by demagnetizing the hot-spot core, while increasing magnetizations at the edge and near regions of large heat loss.
\end{abstract}

DOI: 10.1103/PhysRevLett.118.155001

Inertial confinement fusion (ICF) experiments of indirectly driven hohlraum implosions of plastic capsules at the National Ignition Facility (NIF) are considered. These experiments have observed larger ion temperatures than calculated by fully integrated three-dimensional simulations [1]. Possible explanations are poor modeling of perturbation sources, unstagnated plasma Doppler-shifting the neutron spectra, electron-ion equilibration rates, and self-generated magnetic fields [1]. Applied magnetic fields have been shown to reduce hot-spot thermal losses and increase temperatures in various ICF contexts [2-5]. Self-generated magnetic fields have been measured in the ablation region of ICF experiments [6,7] and field generation and amplification has been studied using simulations of single Rayleigh-Taylor spikes [8,9]. Magnetic transport at the edge of direct-drive hot spots has also been studied in two-dimensional simulations [10]. This letter incorporates self-generated fields into integrated threedimensional simulations of the stagnation phase of indirect-drive targets, modeling self-generated magnetic fields at the hot-spot edge affecting overall target performance through lowered thermal conduction. Nernst magnetic field advection and Righi-Leduc heat flow are, however, found to qualitatively change the hot-spot energy balance and, for the particular multimode case considered here, lower the inferred ion temperature relative to the case where magnetic fields are ignored.

Magnetic fields in a magnetohydrodynamic (MHD) plasma obey [11]

$$
\begin{aligned}
\frac{\partial \underline{B}}{\partial t}= & \nabla \times(\underline{v} \times \underline{B})-\nabla \times \frac{\underline{j} \times \underline{B}}{n_{e} e}-\nabla \times \frac{\underline{\underline{\alpha}} \underline{j}}{n_{e}^{2} e^{2}} \\
& +\nabla \times \frac{\underline{\beta} \bar{\nabla} T_{e}}{e}+\nabla \times \frac{\nabla P_{e}}{n_{e} e},
\end{aligned}
$$

where $\underline{\alpha}$ and $\beta$ are tensor transport coefficients [12], and $\underline{v}$ is the plasma bulk velocity.

The only source of magnetic field in the above equation is the last term, the Biermann battery term; without this, targets with no externally applied magnetic field remain unmagnetized for the duration of the implosion. Assuming the plasma is an ideal gas, the Biermann term can be rearranged to $\left(\nabla T_{e} \times \nabla \ln n_{e}\right) / e$, which makes the analysis of simulation results more intuitive. For a symmetric implosion, the electron density gradients and electron temperature gradients are antiparallel, resulting in no magnetic field. In practice, however, both low- and high-mode perturbations are observed in NIF experiments [1]. Once a perturbation forms, magnetic fields develop. For example, a cold spike pushing into an otherwise symmetric hot-spot cools the plasma near the spike's tip rapidly. This gives rise to nonantiparallel gradients, generating magnetic flux wrapped around the spike.

The other key magnetic transport phenomena in this problem are magnetic field advection (magnetic fields moving with the plasma flow) and the Nernst term (magnetic fields moving down temperature gradients). The Nernst term can be recast as a magnetic field advection velocity [13],

$$
\underline{v}_{N}=-\frac{\beta \wedge \nabla T_{e}}{m_{e} \omega_{e}}
$$

where $\omega_{e}$ is the electron gyrofrequency. $\beta_{\wedge}$ is dependent on the atomic number of the plasma and $\omega_{e} \tau_{e}$, the Hall parameter, where $\tau_{e}$ is the electron-ion collision time. The Hall parameter serves as a useful measure of the plasma magnetization [14],

$$
\omega_{e} \tau_{e}=1.21 \times 10^{16} \frac{T_{e}^{3 / 2}|\underline{B}|}{n_{e} \lambda Z_{\mathrm{eff}}} .
$$


$\lambda$ is the Coulomb logarithm, and $Z_{\text {eff }}=\left\langle Z^{2}\right\rangle /\langle Z\rangle$ where $Z$ is the ionization state and the average is over the ion species. The units are SI, apart from the temperature in $\mathrm{eV}$. Quasineutrality is assumed.

As the Hall parameter increases, thermal conductivities become anisotropic and the conductive heat flow obeys

$\underline{q}=-\kappa_{/ /} \hat{b}\left(\hat{b} \cdot \nabla T_{e}\right)-\kappa_{\perp} \hat{b} \times\left(\nabla T_{e} \times \hat{b}\right)-\kappa_{\wedge} \hat{b} \times \nabla T_{e}$,

where $\hat{b}$ is the magnetic field unit vector. Parallel to the magnetic field $\left(\kappa_{/ /}\right)$there is no conductive modification due to fields. However, as the magnetic fields in this problem are produced perpendicular to $\nabla T_{e}$, this term is usually small. In the perpendicular direction, $\kappa_{\perp}$ decreases with increasing magnetization. Equation (3) shows that magnetization is dependent on the electron temperature and electron density of the plasma, as well as the magnetic field strength. Cold, dense fuel is hard to magnetize and hot, low-density fuel requires relatively small fields to reduce the perpendicular thermal conductivities.

The final term in Eq. (4) is the Righi-Leduc term, which deflects heat flow along isotherms. This term can be large in a plasma with Biermann battery generated magnetic fields, as the fields are generated perpendicular to $\nabla T_{e}$. In a Deuterium-Tritium (DT) plasma, Righi-Leduc heat flow has the greatest magnitude at a Hall parameter of 1 , where approximately $1 / 3$ of the unmagnetized heat flow is directed down the driving temperature gradient, $1 / 3$ deflected along isotherms, and the final $1 / 3$ is fully suppressed. For $\omega_{e} \tau_{e}>1$, the Righi-Leduc heat flow is larger than $\underline{q}_{\perp}$ (the heat flow associated with $\kappa_{\perp}$ ), although both terms tend to 0 for large magnetizations.

This Letter initially outlines the simulation code. Then, results of a $P 2$ perturbation are shown, where the simple topology allows the field generation and transport to be clearly explored. After this, a simulation of a target with single-mode Rayleigh-Taylor spikes penetrating deep into the hot spot is analyzed, showing the effect of Righi-Leduc heat transport. Finally, a realistically perturbed multimode simulation is presented, resulting in large magnetizations.

Following a similar method to that used in $[15,16]$, the simulations presented here are initialized using the onedimensional Chimera code with multigroup radiation transport, providing data at peak radiation temperature to run the three-dimensional Gorgon code for the stagnation phase. Low-mode perturbations are reconstructed from many onedimensional Chimera runs of differing drive intensity, while multihigh-mode perturbations use velocity perturbations that recreate the structure of Rayleigh-Taylor spikes [17].

Gorgon $[18,19]$ is a three-dimensional explicit Cartesian Eulerian code with extended MHD, which includes magnetic transport by bulk and Nernst advection, resistive diffusion, and Biermann battery generated magnetic fields. The Biermann term uses the scheme developed by Graziani [20] (adapted to a magnetic vector potential solver) to enable convergence with cell resolution, preventing the socalled Biermann catastrophe. The Biermann term is turned on after the first shock hits the axis, at $16.0 \mathrm{~ns}$. The Hall term [second on the right-hand side of Eq. (1)] is negligible, along with the $\alpha_{\wedge}, \beta_{/ /}$, and $\beta_{\perp}$ components of $\underline{\alpha}$ and $\underline{\beta}$. The grid size used is $1 \mu \mathrm{m}$.

The electron thermal conduction is an explicit centeredsymmetric fully anisotropic [21] superstepping algorithm [22], allowing heat conduction along magnetic field lines with small amounts of numerical diffusion. This algorithm has been extended to include Righi-Leduc heat flow, calculating the heat flow at cell vertices before resolving onto the faces. Ion thermal conduction is included (although the scheme is isotropic, due to the lower magnetization of the heavier ions). All simulations in this work neglect nonlocal transport, employing a local flux-limited heat flow model, which uses the harmonic mean of the Spitzer heat flow and a factor, $f=0.1$, of the free-streaming limit, $E_{e} v_{t h}$.

The Ettingshausen magnetized thermal transport term [11] is found to be small. For simplicity, an instantaneous local $\alpha$-deposition model is used [23].

Simulations in this Letter are based on shot N130927 from the high-adiabat campaign [24]. The unperturbed chimera neutron yield is $2.0 \times 10^{17}$, which matches calculations by [1]. Perturbations are then added in Gorgon to obtain a neutron yield comparable to experiments [1]. Over the full range of MHD physics packages included in these simulations, the ion temperature for shot N130927 varies between 3.9 and $4.4 \mathrm{keV}$, while the yield is between $4.8 \times 10^{15}$ and $6.1 \times 10^{15}$. The experiment measured $4.5 \pm 0.15 \mathrm{keV}$ and $4.5 \pm 0.1 \times 10^{15}$, while simulations by Clark et al. give $3.9 \mathrm{keV}$ and $3.1 \times 10^{15}$ [1].

Positive $P 2$ perturbations form from low-mode radiation drive asymmetries, causing faster implosion velocities perpendicular to the perturbation axis. Because the capsule is driven harder around the waist, two counterpropagating hot-spot jets form, pointing along the axis from the target center. The implosion is symmetric around the axis of the perturbation.

Figure 1 shows a $P 2$ stagnation-phase simulation, which matches the experimental yield, at two different times. Early on, the hot spot is prolate and $\nabla T_{e}$ is predominantly radially inward, which is antiparallel with the density gradient. However, because the ends of the hot spot are less compressed and have a greater surface area for conductive heat transfer, the ends are cooler than the center and there is a component of the global temperature gradient pointing along the axis of the perturbation. The temperature gradient perpendicular to the density gradient $\left[\nabla T_{e \perp}\right.$ in Fig. 1(a)] changes sign at the center, resulting in opposing magnetic field loops being generated on either end of the hot spot. Most of the magnetic flux is generated at the hotspot edge, where the $\nabla n_{e}$ is the steepest.

The Nernst term advects the magnetic field down electron temperature gradients and out of the hot spot. At this early time, $\nabla T_{e}$ and $\nabla n_{e}$ are almost antiparallel, meaning that as the Nernst term moves the magnetic field, the plasma density increases significantly, lowering the 
(a) Early Time: $16.4 \mathrm{~ns}$

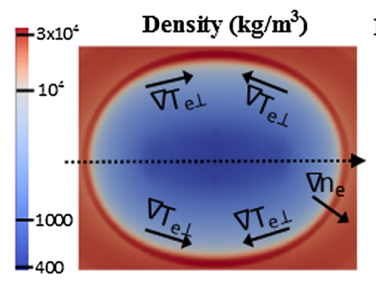

Electron Temperature (ev)

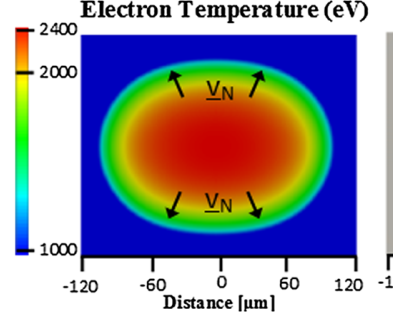

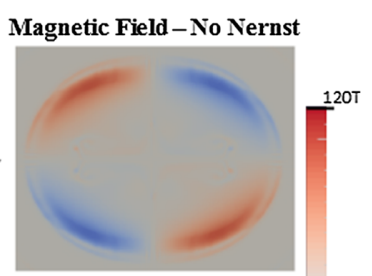

Magnetic Field - Nernst

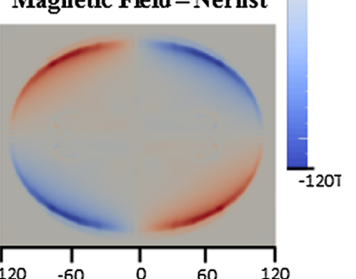

(b) Late Time: $16.7 \mathrm{~ns}$
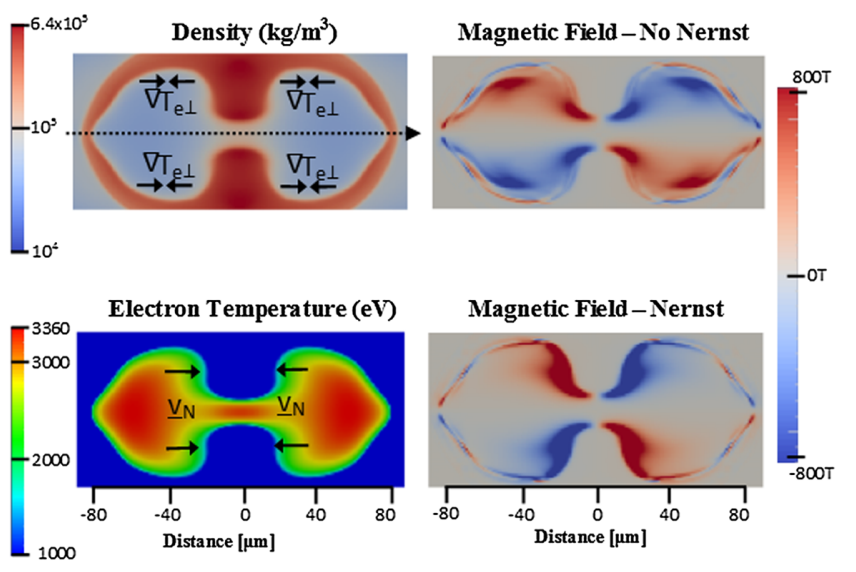

FIG. 1. P2 simulation at two times, comparing magnetic field structure with and without Nernst advection. Initially the Nernst term compresses the field on the outer edge of the hot spot, which is hard to magnetize. Later, cold fuel constricting around the origin pulls in magnetic field from the surrounding region through the Nernst term, which helps thermally isolate the hot spot. $\nabla n_{e}$, Nernst velocities, and $\nabla T_{e \perp}$ are shown for reference, where $\nabla T_{e \perp}$ is the component of $\nabla T_{e}$ perpendicular to $\nabla n_{e}$. Positive magnetic field points out of the page. The dashed arrow represents the axis of symmetry, which is aligned with the hohlraum axis.

resultant magnetization. The Nernst advection velocity increases steadily outwards from the center of the hot spot [because the magnitude of $\nabla T_{e}$ increases; see Eq. (2)] up to a maximum of $100 \mu \mathrm{m} / \mathrm{ns}$ and then decreases rapidly at the outer edge of the hot spot (because the electron-ion collision time decreases), resulting in larger peak magnetic field strengths in the cold dense region outside the hot spot.

Later in time, a contraction of cold dense fuel perpendicular to the axis around the target center causes large heat flows. Figure 1(b) shows that later in time the magnetic field is swept by the Nernst term from a large region of the hot spot towards the constricting cold, dense fuel. This direction is not always antiparallel with the electron density gradient, lowering the magnetization less efficiently than seen at earlier times. Also, as there is a large region producing magnetic flux with Nernst velocities pointing towards the cold fuel constriction, larger peak fields are reached. Adding to this, the cold fuel spike partly thermalizes with its surroundings, resulting in a shallower temperature gradient, which, along with the lower electronion collision time, produces a lower Nernst velocity (up to $20 \mu \mathrm{m} / \mathrm{ns})$. All of this means that in the region either side of the cold fuel pushing into the hot spot, magnetic flux spends more time in hot, low density plasma that is easy to magnetize and therefore the magnetic fields have more of an effect on the thermal conduction than at earlier times. As the cold dense fuel constriction is the main source of thermal conduction out of the hot spot, the Nernst term is actually improving thermal insulation at this time.

Figure 2 shows simulation results of a hot spot with 42 equally spaced single-mode Rayleigh-Taylor cold fuel spikes, which is an informative test problem for RighiLeduc thermal transport. In an experiment, a spike like these could form from a fill tube perturbation.
Self-generated magnetic fields wrap around each cold spike, reducing the thermal conduction from the hot spot, resulting in steep temperature gradients at the interface. Ablative stabilization is reduced [25], enabling the spikes to propagate deeper. Although this deforms the hot spot and brings the cold fuel spikes into contact with the hottest plasma in the core, without Righi-Leduc heat flow the overall effect of the self-generated magnetic fields is still to thermally insulate the hot spot and the average temperature of the hot spot increases (along with the yield).

Figure 2 shows the difference the Righi-Leduc term makes to the total heat flow vector. Additional heat is diverted along the spikes towards their base by the magnetic field. Although the Righi-Leduc heat flow is along isotherms, there is a nonzero divergence of the heat flow and therefore regions gaining and losing energy. The spike tips are cooled by the Righi-Leduc term (counteracting the $\underline{q}_{\perp}$ heat flow into them), lowering the ablative stabilization and allowing the perturbations to penetrate even further into the hot spot. The heat is deposited in regions at the base of the spikes with no magnetic field, allowing heat to diffuse quickly into the cold fuel.

In total, Righi-Leduc heat flow reduces the yield by $9 \%$ compared to the case ignoring self-generated fields. While the concept of magnetic fields reducing thermal containment is counterintuitive, it is precisely because the cold spikes do not thermalize that the Righi-Leduc term can continue to move such significant amounts of heat.

High-mode perturbations generate considerably larger magnetic fields than low modes, as they cause the edge of the hot spot to be larger in area and result in greater temperature gradients perpendicular to the electron density gradients. These magnetic fields are, however, localized on the surface of the hot spot, which is hard to magnetize. 


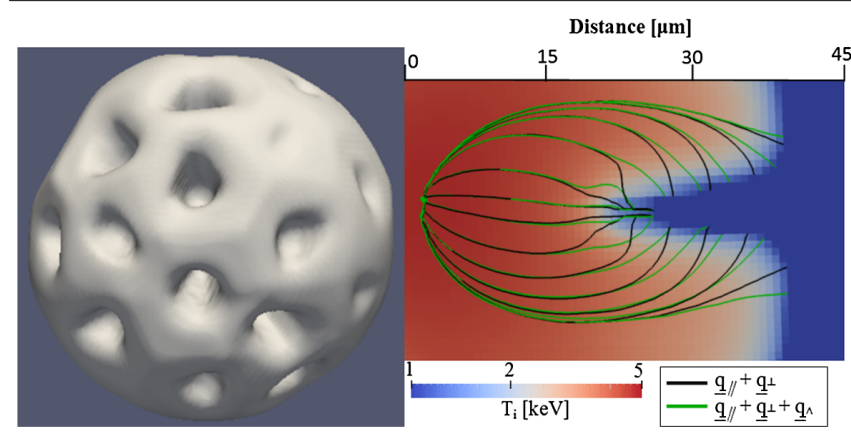

FIG. 2. Left: hot-spot surface regularly perturbed by single mode-number cold fuel spikes. Right: two-dimensional ion temperature slice of one spike, with the $\left(\underline{q}_{/ /}+\underline{q}_{\perp}\right)$ and $\left(\underline{q}_{/ /}+\right.$ $\left.\underline{q}_{\perp}+\underline{q}_{\wedge}\right)$ vectors overlain, where $\underline{q}_{/ /}$is the heat flow parallel to the magnetic field lines, $\underline{q}_{\perp}$ is the heat flow down the temperature gradient perpendicular to the magnetic field, and $\underline{q}_{\wedge}$ is the RighiLeduc heat flow. The Righi-Leduc heat flow moves heat away from the tip of the spike towards the base.

When low-mode perturbations are also present, the hot-spot surface is pushed into the hot core of the target, causing the plasma to become significantly magnetized.

Figure 3 shows simulation results at peak neutron time for a combination of low- and high-mode perturbations [16], with self-generated magnetic field strengths reaching over 10,000 T. It can be seen that the largest plasma magnetizations are reached in the regions where cold spikes have pushed the magnetic fields into the core of the hot spot, resulting in peak local Hall parameters greater than 3 and $\kappa_{\perp} / \kappa_{/ /}<0.1$ in some locations. Until peak neutron time, the average magnetization in the $1 \mathrm{keV}$ hot spot increases, because more fields are generated and hydrodynamically compressed. Then, as the hot spot cools and becomes more dense, the plasma demagnetizes.

Ion temperatures are inferred from the time-dependent energy spectra of unscattered DT neutrons $[16,26]$. Table I compares the capsule performance for different levels of MHD effects incorporated.

As seen in the $P 2$ simulation, Nernst magnetic field advection demagnetizes the inner core of the hot spot, increasing the field strength at the edge. Early in the burn pulse this results in the heat flow being less magnetized. Later, as large cold spikes propagate deep into the hot spot (for example, coming from the top of Fig. 3) the Nernst term advects more field to these regions of large heat loss and increases the magnetization. When the hot spot demagnetizes after peak neutron output, the large temperature gradients at the edge of the hot spot (which were sustained by Nernst advection increasing the magnetization locally earlier in time) result in greater heat flows. In this case, the overall effect of Nernst advection is to lower the yield and ion temperature.

Without Righi-Leduc heat flow, $\kappa_{\perp} / \kappa_{/ /}$can be as low as 0.02 , corresponding to a magnetization larger than 7.0. Conductive losses from the $1 \mathrm{keV}$ hot spot are reduced by

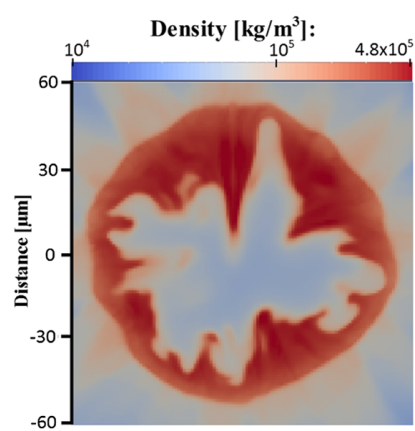

Electron Temperature $[\mathrm{eV}]:_{6000}$
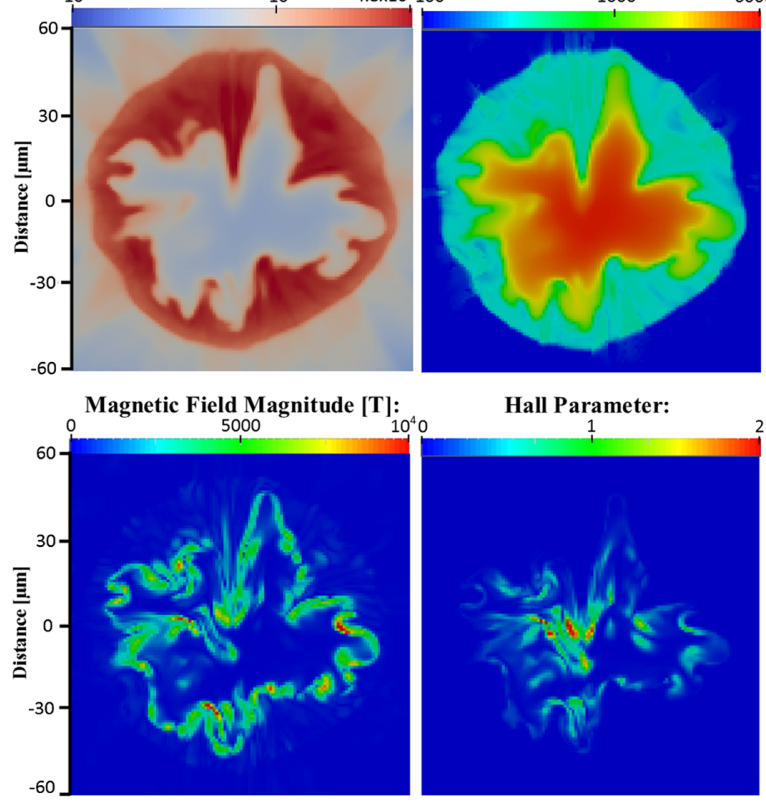

FIG. 3. Two-dimensional slices at neutron bang time of a simulation with a combination of high- and low-mode perturbations.

$30 \%$ in the period leading up to peak neutron output. Magnetization of the spikes lowers ablative stabilization, allowing the cold fuel to propagate further into the hot spot. However, the reduction in thermal conduction still increases the hot-spot energy containment significantly.

With Righi-Leduc included, the magnetized temperature falls below the unmagnetized case. This happens through the same mechanism discussed earlier in the single-mode test problem. While the magnetic fields reduce the amount of conduction into the low-mode spikes, the resultant steep gradient provides a platform from which the Righi-Leduc component deflects heat along isotherms around the outside of the spikes towards their base. Therefore, the cold spikes' tips are cooled, reducing ablative stabilization and allowing spike propagation further into the hot-spot core. This heat is then dumped at the base of the spike, which can conduct into the cold, dense fuel effectively. The average magnetization of the hot spot is reduced by a factor of 2 by the Righi-Leduc term.

TABLE I. Summary of multimode results for different MHD packages.

\begin{tabular}{lccc}
\hline \hline MHD packages & $\begin{array}{c}\text { Neutron } \\
\text { yield }\end{array}$ & $\begin{array}{c}T_{i} \\
{[\mathrm{keV}]}\end{array}$ & $\begin{array}{c}\text { Minimum } \\
\kappa_{\perp} / \kappa_{/ /}\end{array}$ \\
\hline None & $5.43 \times 10^{15}$ & 4.0 & 1.00 \\
Biermann & $6.63 \times 10^{15}$ & 4.4 & 0.01 \\
Biermann + Nernst & $6.10 \times 10^{15}$ & 4.2 & 0.02 \\
$\begin{array}{c}\text { Biermann + Nernst } \\
\quad \text { Righi-Leduc }\end{array}$ & $4.80 \times 10^{15}$ & 3.9 & 0.10 \\
\hline \hline
\end{tabular}


The magnetic fields calculated here lower the Larmor radius of fusion $\alpha$ particles to as low as $20 \mu \mathrm{m}$, comparable with the hot-spot radius. However, these field strengths are only seen in boundary regions smaller than $20 \mu \mathrm{m}$, meaning full containment of $\alpha$ particles is not expected. It is possible that magnetic fields change the nature of the fire polishing effect [27], deflecting $\alpha$ particles away from the cold fuel spikes.

In summary, self-generated magnetic fields in the stagnation phase of ICF implosions have been shown to cause significant magnetizations when low- and high-mode perturbations are both present. Although heat flow down temperature gradients is reduced, the Righi-Leduc heat flow effectively pumps heat around the low-mode perturbations without thermalizing the driving temperature gradient, cooling the hot spot.

The Nernst effect is found to qualitatively change the magnetization of the hot spot by demagnetizing the hot core, while increasing magnetization at the edge of the hot spot around cold Rayleigh-Taylor spikes that inject late into the hot core.

If perturbation amplitudes are increased, the magnetic flux produced increases and larger volume-averaged magnetizations are reached earlier in time, although the magnetization decays faster as the hot spot cools.

The relative importance of each magnetized transport effect is expected to vary with drive profile and perturbation combination. This is the subject of future work.

It is clear that accurate prediction of ICF hot-spot degradation requires the consideration of magnetic field effects. As target designs move closer to ignition and there is more self-heating of the fuel, magnetization contributions are expected to increase considerably, potentially leading to a thermally isolated hot spot (the Righi-Leduc term decreases in magnitude for large magnetizations). On the other hand, if the implosions become more symmetric, lower fields are generated and magnetization effects are less important.

Further work is required in modeling the effect of magnetic fields on $\alpha$-particle transport, which could modify the propagation of a burn wave.

The results reported in this Letter were obtained using the United Kingdom National Supercomputing Service ARCHER and the Imperial College High Performance Computer Cx1. This work was supported by the Engineering and Physical Sciences Research Council through Grants No. EP/K028464/1 and No. EP/ L000237/1 and by AWE Aldermaston.

*.walsh14@imperial.ac.uk

${ }^{\dagger}$ Present address: First Light Fusion, 10 Oxford Industrial Park, Mead Road, Yarnton, Kidlington OX5 1QU, United Kingdom.

[1] D. S. Clark, C. R. Weber, J. L. Milovich, J. D. Salmonson, A. L. Kritcher, S. W. Haan, B. A. Hammel, D. E. Hinkel,
O. A. Hurricane, O. S. Jones, M. M. Marinak, P. K. Patel, H. F. Robey, S. M. Sepke, and M. J. Edwards, Phys. Plasmas 23, 056302 (2016).

[2] P. Y. Chang, G. Fiksel, M. Hohenberger, J. P. Knauer, R. Betti, F. J. Marshall, and D. D. Meyerhofer, Phys. Rev. Lett. 107, 035006 (2011).

[3] S. A. Slutz and R. A. Vesey, Phys. Rev. Lett. 108, 025003 (2012).

[4] P. F. Schmit et al., Phys. Rev. Lett. 113, 155004 (2014).

[5] I. R. Lindemuth and R. C. Kirkpatrick, Nucl. Fusion 23, 263 (1983).

[6] M. J.-E. Manuel, C. K. Li, F. H. Séguin, J. Frenje, D. T. Casey, R. D. Petrasso, S. X. Hu, R. Betti, J. D. Hager, D. D. Meyerhofer, and V. A. Smalyuk, Phys. Rev. Lett. 108, 255006 (2012).

[7] I. V. Igumenshchev, A. B. Zylstra, C. K. Li, P. M. Nilson, V. N. Goncharov, and R. D. Petrasso, Phys. Plasmas 21, 062707 (2014).

[8] B. Srinivasan and X.Z. Tang, Phys. Plasmas 19, 082703 (2012).

[9] F. Modica, T. Plewa, and A. Zhiglo, High Energy Density Phys. 9, 767 (2013).

[10] A. Hata, K. Mima, A. Sunahara, H. Nagatomo, and A. Nishiguchi, Plasma Fusion Res. 1, 020 (2006).

[11] S. I. Braginskii, Rev. Plasma Phys. 1, 205 (1965).

[12] E. M. Epperlein and M. G. Haines, Phys. Fluids 29, 1029 (1986).

[13] A. Nishiguchi, T. Yabe, M. G. Haines, M. Psimopoulos, and H. Takewaki, Phys. Rev. Lett. 53, 262 (1984).

[14] J. D. Huba, NRL Plasma Formulary (Naval Research Laboratory, Washington, DC, 2013).

[15] S. Taylor and J. P. Chittenden, Phys. Plasmas 21, 062701 (2014).

[16] J. P. Chittenden, B. D. Appelbe, F. Manke, K. McGlinchey, and N. P. L. Niasse, Phys. Plasmas 23, 052708 (2016).

[17] D. Layzer, Astrophys. J. 122, 1 (1955).

[18] J. P. Chittenden, S. V. Lebedev, C. A. Jennings, S. N. Bland, and A. Ciardi, Plasma Phys. Controlled Fusion 46, B457 (2004).

[19] A. Ciardi, S. V. Lebedev, A. Frank, E. G. Blackman, J. P. Chittenden, C. J. Jennings, D. J. Ampleford, S. N. Bland, S. C. Bott, J. Rapley, G. N. Hall, F. A. Suzuki-Vidal, A. Marocchino, T. Lery, and C. Stehle, Phys. Plasmas 14, 056501 (2007).

[20] C. Graziani, P. Tzeferacos, D. Lee, D. Q. Lamb, K. Weide, M. Fatenejad, and J. Miller, J. Phys. Conf. Ser. 719, 012018 (2016).

[21] P. Sharma and G. W. Hammett, J. Comput. Phys. 227, 123 (2007).

[22] C. D. Meyer, D. S. Balsara, and T. D. Aslam, Mon. Not. R. Astron. Soc. 422, 2102 (2012).

[23] G. S. Fraley, Phys. Fluids 17, 474 (1974).

[24] O. A. Hurricane et al., Nature (London) 506, 343 (2014).

[25] M. A. Partha, S. W. Haan, J. Koning, M. M. Marinak, C. R. Weber, and D. S. Clark, in Simulated Impact of Self-Generated Magnetic fields in the Hot Spot of NIF Implosions (APS DPP, San Jose, 2016).

[26] B. Appelbe and J. Chittenden, High Energy Density Phys. 11, 30 (2014).

[27] H. Takabe and T. Ishii, Jpn. J. Appl. Phys. 32, 5675 (1993). 